\title{
Comparison of crystallization characteristics and mechanical properties of polypropylene processed by ultrasound and conventional micro injection molding
}

\author{
Davide Masato $^{\mathrm{a}, \mathrm{b}, \dagger}$, Maksims Babenko ${ }^{\mathrm{a}}$, Banah Shriky ${ }^{\mathrm{a}}$, Tim Gough ${ }^{\mathrm{a}}$, Giovanni Lucchetta ${ }^{\mathrm{b}}$, Ben \\ Whiteside ${ }^{\mathrm{a}}$ \\ ${ }^{a}$ Faculty of Engineering and Informatics, University of Bradford, Bradford, UK \\ ${ }^{\mathrm{b}}$ Department of Industrial Engineering, University of Padova, via Venezia 1, Padova, Italy \\ † corresponding author, davide.masato@phd.unipd.it
}




\title{
Comparison of crystallization characteristics and mechanical properties of polypropylene processed by ultrasound and conventional micro injection molding
}

Davide Masato, Maksims Babenko, Banah Shriky, Tim Gough, Giovanni Lucchetta, Ben Whiteside

\begin{abstract}
Ultrasound injection molding has emerged as an alternative production route for the manufacturing of micro scale polymeric components, where it offers significant benefits over the conventional micro injection molding process. In this work, the effects of ultrasound melting on the mechanical and morphological properties of micro polypropylene parts were characterized. The ultrasound injection molding process was experimentally compared to the conventional micro injection molding process using a novel mold, which allows mounting on both machines and visualization of the melt flow for both molding processes. Direct measurements of the flow front speed and temperature distributions were performed using both conventional and thermal high-speed imaging techniques. The manufacturing of micro tensile specimens allowed the comparison of the mechanical properties of the parts obtained with the different processes. The results indicated that the ultrasound injection molding process could be an efficient alternative to the conventional process.
\end{abstract}

Keywords: ultrasound injection molding; micro injection molding; mechanical properties; morphology; flow visualization 
In microsystems technology, polymer micro components have been attracting large attention for several applications, such as micro electronics, micro mechanics, micro optics and micro biomedical devices [1-5]. However, the increasing market trend needs to be sustained by the development of effective manufacturing processes that could support their mass production [6,7].

Micro injection molding ( $\mu \mathrm{IM})$ is one of the most popular manufacturing technologies used to produce thermoplastic micro components, which typically have sub-100 mg masses and dimensions or tolerances in the micrometer range $[8,9]$. The process depends fundamentally on the molten polymer accurately replicating the mold geometry during the mold filling and cooling phases through a complex mechanism [10], which is determined by the complicated relationship between materials properties, processing conditions and mold geometry [11-13].

Despite the significant improvements and the ongoing research, the quality of $\mu \mathrm{IM}$ parts is still hindered by some intrinsic process limitations. Lucchetta et al. investigated the technological limits of $\mu \mathrm{IM}$ observing that the achievement of optimum quality of the molded parts is still challenging, even using state-of-the-art $\mu \mathrm{IM}$ process technology (i.e. rapid heat cycle molding and cavity air evacuation) [14]. Indeed, the uneven distribution of cavity pressure hindered the achievement of a complete and homogenous replication of a high aspect ratio micro-structured surface. In fact, the filling phase of the $\mu \mathrm{IM}$ process is characterized by highly shear-stressed melt along the flow direction, which leads to anisotropy in the properties of the final molded parts [15].

Moreover, the $\mu \mathrm{IM}$ process is significantly affected by complex boundary conditions due to the marked thermal gradients and the typically very small cavity thicknesses [16]. These conditions affect the formation of the 'skin-core' morphology and are the cause for the nucleation and the formation of smaller and more oriented crystalline structures within the molded parts [17]. 
In this context, some new process concepts have emerged aiming at improving processing performances and the quality of the molded micro parts. In particular, ultrasound injection molding (usIM) was proposed due to the interesting benefits it could yield compared to the conventional $\mu \mathrm{IM}$ process [18].

The usIM process is characterized by the use an ultrasound horn for the melting of the polymer pellets [19]. The energetic ultrasound waves are exploited to cyclically heat and deform the polymer at ultrasound frequencies. The melting of the polymer is then controlled by the friction heating at the contact points between the pellets and by the viscoelastic heating generated by the damping of the ultrasonic oscillation within the polymer itself $[20,21]$.

A conventional injection molding plastication unit typically comprises of a reciprocating screw and a barrel with electrical heaters. The energy required to heat a volume of material for a single shot is relatively high due to heat losses to the environment and the heating of materials that will not contribute to the manufactured components (including waste materials generated during purging and the heat required to bring the barrel and screw to the set melt temperature) [18]. In ultrasound injection molding, significantly lower energy is required for the melting of the polymer due to the absence of electrical heaters and no requirement to pre-heat the components of the machine. Moreover, with ultrasound melting there is no material residence time and consequent degradation, which is particularly important for temperature sensitive polymers. In addition, the ultrasound melting energy is provided only to the pellets that are in contact with the sonotrode, thus for each molding cycle only the material required for the single shot is melted [19].

Other advantages of the usIM process include the reduction of material wastage, which is particularly important for micro molding applications where the size of the parts can be significantly smaller than the runners and the polymers can be very expensive (e.g. biomedical applications). 
Moreover, no purging is required for material changes and cleaning, hence reducing the risk for cross contamination.

The ultrasonic energy can also be sustained during the injection phase in order to continuously provide heat to the polymer during cavity filling and consequently reduce the required injection pressure [22]. Moreover, the sprue acts as an 'energy director' that is able to orientate the ultrasonic waves in the flow direction. The enhanced flowability of the melt polymer can then be exploited to achieve higher molecular chain relaxation and reduced residual stresses in comparison with conventional $\mu \mathrm{IM}[23,24]$.

Despite the significant advantages, the usIM has been poorly investigated in the literature and it is still difficult to control. Sacristán et al. investigated the effects of the ultrasonic vibration on the manufacturing of different parts using polylactide [25]. They have reported that the process requires optimization in order to control material degradation and to avoid incomplete filling of the cavity. Grabalosa et al. successfully manufactured some dumbbell shaped polyamide tensile specimens observing that the combination of vibration amplitude, applied pressure and vibration time determines the amount of ultrasonic energy that is transferred to the polymer pellets [26]. Moreover, they reported that the effect of processing parameters is still unknown and the optimization is complex as it can affect parts filling, morphology and mechanical properties.

The main process parameters are the amplitude of the vibration, the sonicating time and the applied force. Thus, compared to $\mu \mathrm{IM}$, process control for usIM can be significantly different, indicating the necessity of improving the understanding of the process [27]. In this sense, Negre et al. investigated the effects of some process parameters, showing how to approach process optimization (e.g. increased ultrasound time and low injection speed improve the filling) and reporting significant variability in filling length and parts weight [28].

In this work, the effect of ultrasound injection molding on the mechanical properties of polypropylene micro parts produced was investigated. A novel injection molding tool, which allows the 
visualization of the melt flow through a sapphire window, was used to produce micro tensile samples using both conventional and ultrasound injection molding. The processes were analyzed and compared using both conventional and infrared high-speed imaging in order to relate their filling behavior and temperature distribution to parts mechanical and morphological properties.

\section{Materials and methods}

\subsection{Part design}

The part considered in this study is a micro tensile bar, which dimensions were designed according to ISO 527-1 Type 5A and scaled-down by a factor of ten (Fig. 1). In the mold, the cavity was placed on the moving half, at the end of semi-cylindrical cold runner (diameter: $2 \mathrm{~mm}$, length: $16 \mathrm{~mm}$ long).
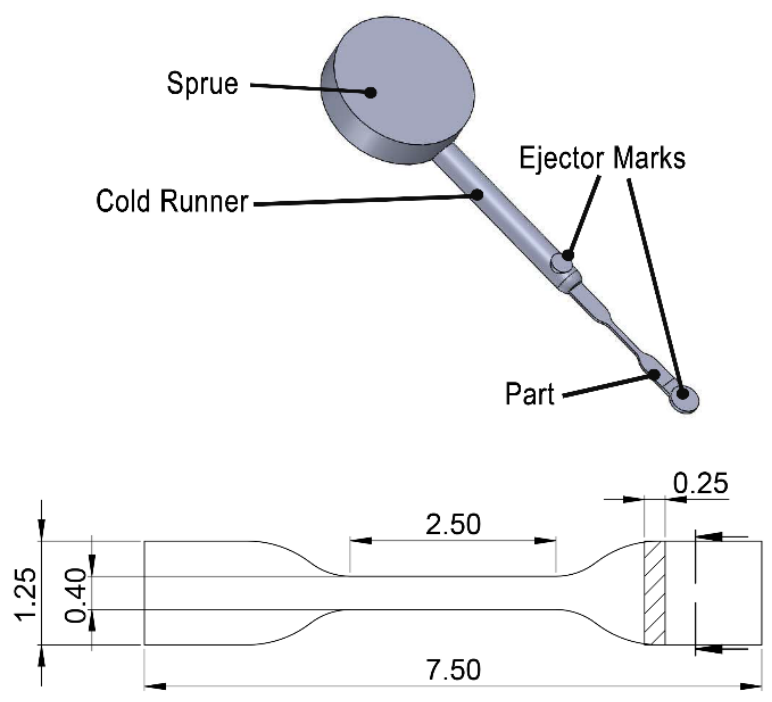

Figure 1. Design of the molded micro part. All dimensions are in millimeters.

A commercial crystalline polypropylene-homopolymer (INEOS PP GA12) was selected for the molding experiments. The polymer is characterized by high flowability, high transparency, good 
dimensional stability and melt flow rate (MFR) consistency, which makes it ideal for micro molding applications. Table 1 reports the main properties of the selected polymer.

Table 1. Main properties of the polymer selected for the molding experiments.

\begin{tabular}{llll}
\hline \multicolumn{1}{c}{ Property } & Units & \multicolumn{1}{c}{$\begin{array}{c}\text { Test } \\
\text { Method }\end{array}$} & Value \\
\hline Density & $\mathrm{g} / \mathrm{cm}^{3}$ & ISO 1183 & 0.93 \\
MFR $\left(230^{\circ} \mathrm{C}-2.16 \mathrm{~kg}\right)$ & $\mathrm{g} / 10 \mathrm{~min}$ & ISO 1133 & 12 \\
Transition Temperature & ${ }^{\circ} \mathrm{C}$ & ISO 6721 & 182 \\
\hline
\end{tabular}

\subsection{Ultrasound injection molding}

An ultrasound vertical molding machine (Sonorus 1G, Ultrasion S.L.) was used for the ultrasound injection molding (usIM) experiments. The machine is characterized by an ultrasound horn that is used to melt the polymer by exploiting the energy transmitted to the polymer through the ultrasonic vibrations. A digital ultrasound generator (Branson, 50DCXs30VRT) controls the amplitude and the duration of the vibration, with a frequency of $30 \mathrm{kHz}$ and a maximum power of $1.5 \mathrm{~kW}$. The piezoelectric transducer is used to convert high-frequency electrical signals into a mechanical vibrations.

For each molding cycle, a predefined number of standard polymer pellets is counted by a laser system and dosed into the metering chamber (Fig. 2 (a)). Then, the sonotrode, which is fitted on the upper mold half, moves down to the injection position (Fig. 2 (b)). The injection is carried out by a vertical plunger ( $8 \mathrm{~mm}$ diameter) fitted in the lower half of the mold, which moves upwards pushing the polymer pellets towards the vibrating ultrasound horn. The mechanical vibration melts the polymer granules that are simultaneously injected into the cavity (Fig. 2 (c)). The ultrasonic vibration was sustained for the whole duration of the injection phase. 


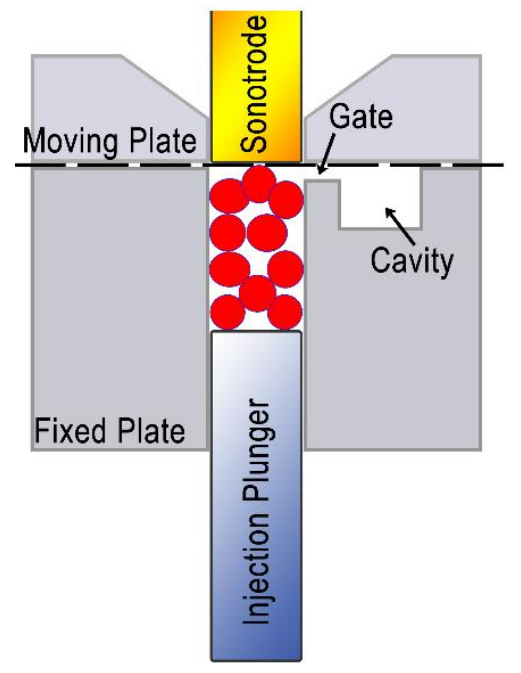

(a)

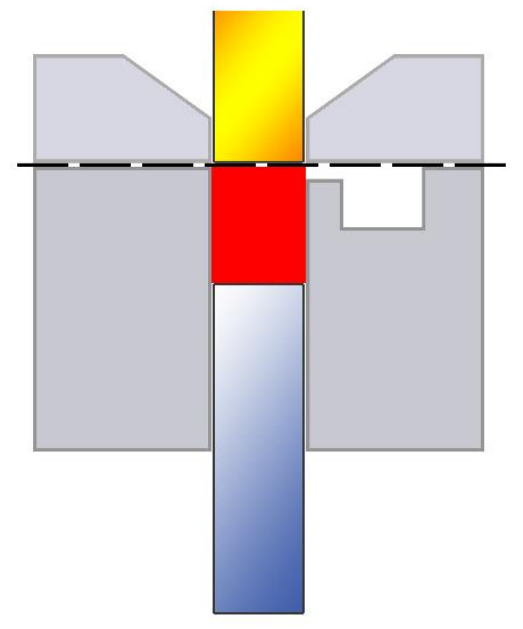

(b)

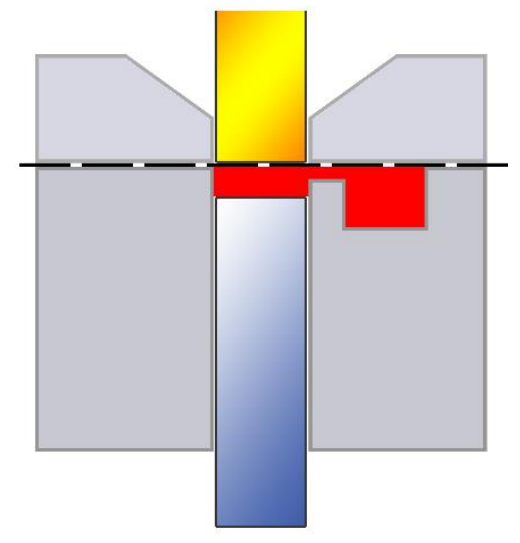

(c)

Figure 2. Main phases of the usIM process, (a) pellet feeding and compression, (b) melting, (c) injection.

\subsection{Micro injection molding}

A state-of-the-art Wittmann Battenfeld, MicroPower 15 electric machine was used for the micro injection molding $(\mu \mathrm{IM})$ experiments. The machine is characterized by the separation of the metering and injection systems: the polymer pellets are melted by the mechanical action of a $14 \mathrm{~mm}$ plasticizing screw and electrical heaters, while injection is performed by a separated $5 \mathrm{~mm}$ injection plunger. The maximum injection speed is of $750 \mathrm{~mm} / \mathrm{s}$, to achieve a maximum injection pressure of $2700 \mathrm{bar}$ and a maximum shot size of $1 \mathrm{~cm}^{3}$.

\subsection{Mold design}

The mold assembly was designed, from a Hasco K-standard modular system, to be mounted on both the usIM and $\mu \mathrm{IM}$ machines. Minor modifications were only introduced to allow coupling with the different injection units. 
The mold was designed to allow the direct visualization of the polymer melt flow during molding cycles on both machines [29]. A sapphire window (diameter: $26.5 \mathrm{~mm}$, thickness: $4 \mathrm{~mm}$ ) was placed on the fixed mold half, as shown in Fig. 3. A rectangular pocket was machined on the clamping plate in order to fit a $45^{\circ}$ tilted first surface gold mirror, which was mounted and aligned with the center of the cavity.

The mold temperature was set using four electrical cartridge heaters, placed in couples in the mold fixed and moving halves together with a thermocouple.

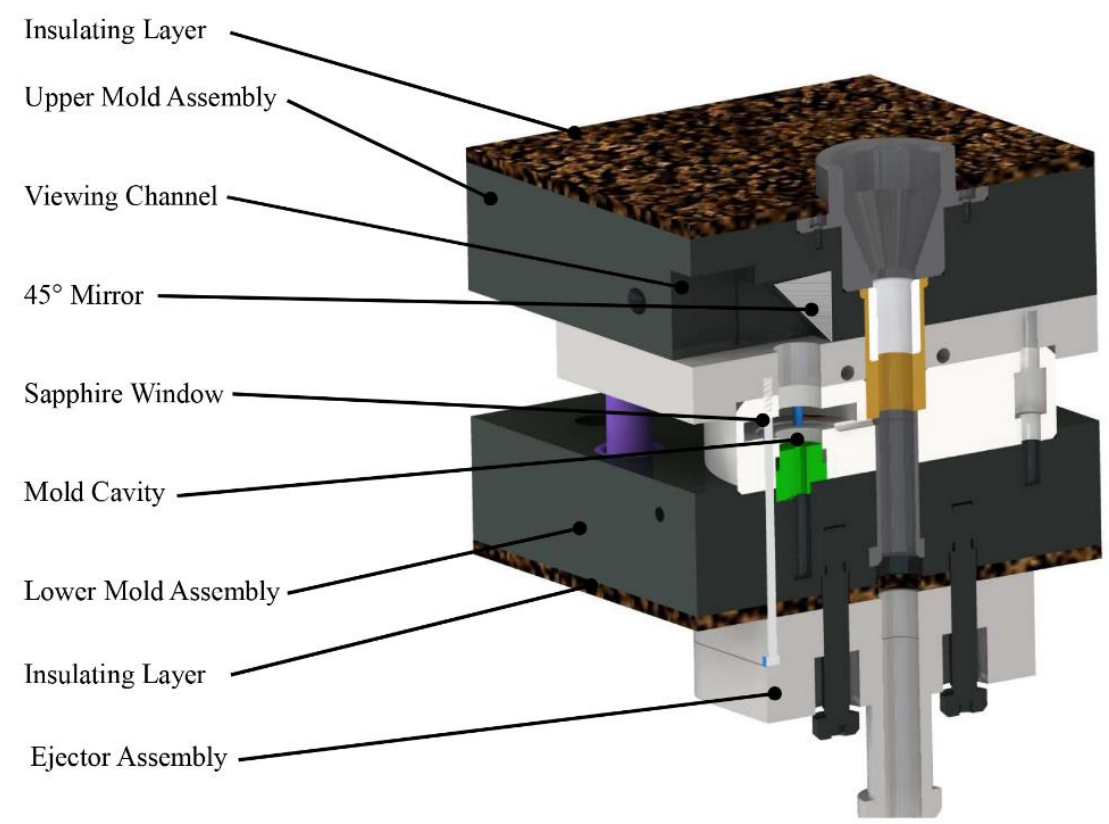

Figure 3. Mold design, as mounted on the usIM machine, and indication of its main components.

\subsection{Experimental approach}

The analysis of the effects of ultrasound melting on the mechanical properties of micro-molded polypropylene parts was approached by direct comparison with conventional $\mu \mathrm{IM}$. The main process 
parameters were set the same in the two experimental setups, considering the literature, recommendations of the material supplier and technological limitations of the machines:

- mold temperature: $80^{\circ} \mathrm{C}$;

- cooling time: $5 \mathrm{~s}$;

- clamping force: $150 \mathrm{kN}$

- $\quad$ set flow rates: 2,$500 ; 5,000 ; 10,000 \mathrm{~mm}^{3} / \mathrm{s}$.

In the usIM machine, for each cycle seven pellets were dosed into the metering chamber $(8 \mathrm{~mm}$ diameter, $6 \mathrm{~mm}$ length) before the beginning of the injection, which was performed with an applied force of $700 \mathrm{~N}$. The ultrasound vibration was controlled by setting an amplitude of 0.9 of the maximum (i.e. $10 \mu \mathrm{m})$ and a sonicating time of 3 seconds.

For the $\mu \mathrm{IM}$ process, the melt temperature was set to $220^{\circ} \mathrm{C}$ and the shot size was fixed to the value of $1.2 \mathrm{~mm}$. The switch-over point was set at the $80 \%$ of the injection pressure peak, while a packing pressure of 500 bar for 3 seconds was applied.

For each one of the molding processes, ten parts were molded with the same set of process parameters and then characterized. In order to achieve adequate stability for both molding processes, 20 molding cycles were performed before the collection of the first part. Then, for each molding technology, the parts for the characterization were collected, one every 5 cycles.

\subsection{Characterization of the molded parts}

\subsubsection{Mechanical properties}

The mechanical behavior of the molded micro parts was characterized by means of uniaxial tensile tests at room temperature using a mechanical testing system (Biomomentum, Mach- $1^{\mathrm{TM}}$ ), equipped with a $25 \mathrm{~N}$ load cell. The molded samples were fixed using custom grips and the tests were performed with a tensile speed of $10 \mathrm{~mm} / \mathrm{min}$. Elastic modulus, stress at break and strain at break were taken into 
consideration for the comparison of the mechanical properties of the samples molded with the two different injection molding technologies.

\subsubsection{Evaluation of crystallinity}

The use of ultrasound to melt the polymer and the consequent filling mechanism can affect the formation of crystalline structure within the solidifying polymer [30]. Thus, the flow-induced crystallization of the molded micro parts was evaluated by means of Differential Scanning Calorimetry (DSC - TA Instruments, Discovery).

Thermal analysis were conducted on samples that were cut from the gauge section of the molded micro tensile specimens. Thermal cycles were conducted with a rate of $10^{\circ} \mathrm{C} / \mathrm{min}$ and by following a heating/cooling/heating procedure, as shown in Fig. 4. Indeed, the molded polymer samples initially present the morphological structure as induced by the processing conditions. Upon the first heating ramp, the sample is melted and in the next cooling and heating cycles it crystallizes at much lower heating rates, resulting in higher crystallinity. The crystallinity induced by injection molding processing was evaluated as the ratio between the enthalpy of the first and second heating cycles. 


\subsubsection{Morphological characterization}

The effect of injection molding processing on the morphology of the polymer parts was characterized using a modular small- (SAXS) and wide-angle (WAXS) x-ray scattering system (AntonPaar, SAXSpace system). An additional hot-pressed PP sample was prepared to compare the scattering results from usIM and $\mu \mathrm{IM}$ experiments to those from a zero shear process.

An x-ray radiation with a wavelength $0.154 \mathrm{~nm}$ was generated at $50 \mathrm{kV}$ and $1 \mathrm{~mA}$. Sample-todetector distances of $112 \mathrm{~mm}$ and $308 \mathrm{~mm}$ were used for WAXS and SAXS measurements, respectively. Debye diffractions were recorded across the whole thickness, twice at each position. Samples were rotated to obtain a full $90^{\circ}$ diffraction of the sample to overcome detector limitation and the beam was fixed in the transverse direction (TD) perpendicular to the flow direction (MD). Scattering data were processed using Anton-Paar's SAXSTreat software. 


\section{Flow visualization setup}

\subsection{High-speed imaging}

The filling behavior in the usIM process was compared to that of the $\mu \mathrm{IM}$ process by direct highspeed imaging of the melt flow. The position of the advancing melt flow front was observed during the processes using a NAC, Memrecam HX-6 camera and a compact telecentric lens (Edmund Optics, Techspec ${ }^{\oplus}$ ) with a magnification $0.75 \mathrm{x}$ and a working distance of $110 \mathrm{~mm}$. The polymer flow was illuminated using a white light LED illuminator (SugarCUBE ${ }^{\mathrm{TM}}$, LED Illuminator) connected to a fiber optic light guide.

Frame rates of 20,000 fps and 5,000 fps were adopted for the $\mu \mathrm{IM}$ and usIM experiments, respectively. The frame size (i.e. field of view) was of 640x 128 pixels and the resolution of 70 pixels $/ \mathrm{mm}$. For both processes, the recording was triggered using a digital signal output from the machine that was synchronous with the initial forward movement of the injection plunger during injection, after the mold closed to the viewing position.

The acquired image sequences were analyzed using Labview 2013® (National Instruments). Specifically, the 'Edge detector' function, implemented in a National Instruments Vision Assistant subVI, was used to automatically determine the coordinates of the flow front position for each one of the acquired frames, as indicated in Fig. 5.

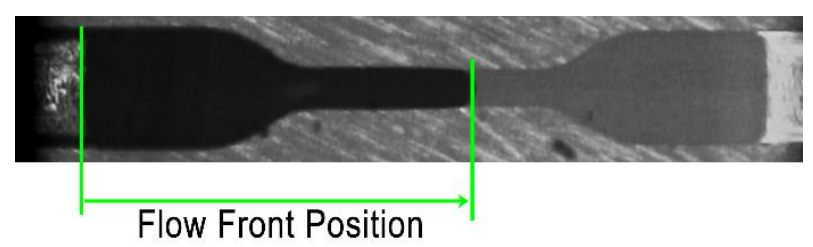

Figure 5. Edge detection of the flow front position on the acquired frame. 


\subsection{Thermal imaging}

The temperature distribution during the filling phase was evaluated using thermal imaging of the melt flow. The thermal analysis of the cavity filling was performed using a high-speed, high-sensitivity infrared camera (FLIR, X6540SC). The camera has 640x512 pixels focal plane array (FPA) detector, operates in the spectral range of 1.5-5.1 $\mu \mathrm{m}$, has pixel pitch of $15 \mu \mathrm{m}$ and aperture of $\mathrm{f} / 3$. Detector sensitivity at $25^{\circ} \mathrm{C}$ (NETD) is smaller than $20 \mathrm{mK}$.

For the usIM experiments frame size of $640 \times 104$ pixels $(9.60 \times 1.56 \mathrm{~mm})$, integration time of $50 \mu \mathrm{s}$ and frame rate of $500 \mathrm{fps}$ were used. A higher frame rate was adopted for the $\mu \mathrm{IM}$ process, due to the faster melt flow velocity. For this reason, the frame size was reduced to 640x8 pixels (through the middle of the cavity) allowing to capture thermal data at the frame rate of 2,500 fps. The integration time was kept unchanged at $50 \mu \mathrm{s}$.

Polymer melts emit thermal radiation energy from their core, both on their surface and subsurface. Thus, radiation characteristics not only depend on cavity geometry (i.e. mainly thickness) but also on melt flow temperature distribution. This makes the polymer semi-transparent in near to mid infrared regions and interpretation of thermo-camera measurements complicated. In this work, the measurement of polymer surface temperature was carried out by compounding the base polypropylene with $4 \%$ (weight) of carbon black (CB) pigment [31], which makes it opaque in the infrared region where the thermo-camera has the highest sensitivity (i.e. 3-5 $\mu \mathrm{m}$ ). Conversely, temperature measurements of unfilled polypropylene (PP) during injection molding processing were considered as volumetric/bulk values. Then, the effect of ultrasound melting on temperature distribution in the melt flow were compared to conventional $\mu \mathrm{IM}$ by setting emissivity to 1 for both unfilled and CB filled PP.

\section{Results of molded parts characterization}

\subsection{Mechanical properties}


The mechanical properties of the molded micro tensile specimens were characterized and the effect of ultrasound melting was evaluated by comparison with parts molded by conventional $\mu \mathrm{IM}$. Fig. 6 shows the results of the tensile strength tests, indicating that the overall mechanical properties achieved with the usIM process are higher than those obtained by $\mu \mathrm{IM}$. Specifically, the parts molded using the usIM machine are characterized on average by a higher value of the maximum tensile strength $(+10 \%)$ compared to the $\mu \mathrm{IM}$ samples. Similarly, the average value of the strain at break was higher $(+12 \%)$ for the samples produced by usIM. The values of the elastic modulus were of similar magnitude comparing the two injection molding technologies.

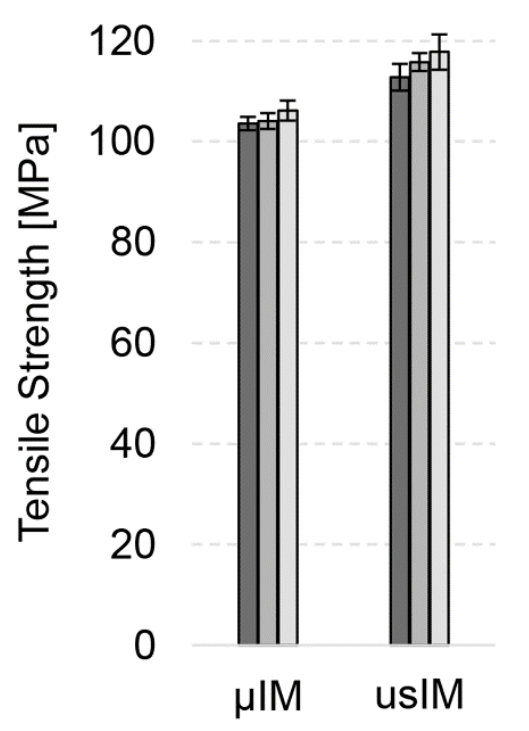

(a)

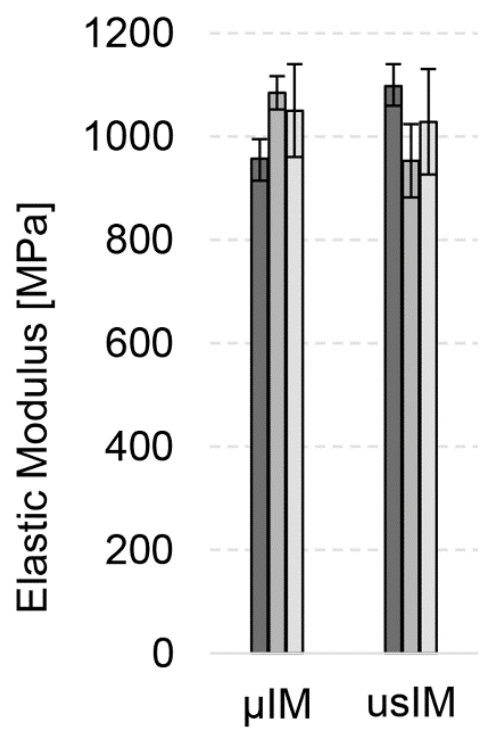

(b)

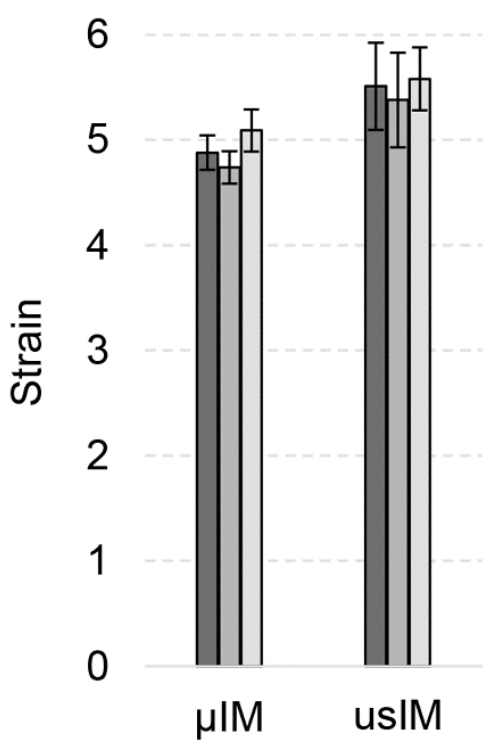

(c)

\section{$\square 2,500 \mathrm{~mm}^{\wedge} 3 / \mathrm{s} \quad \square 5,000 \mathrm{~mm}^{\wedge} 3 / \mathrm{s} \quad \square 10,000 \mathrm{~mm}^{\wedge} 3 / \mathrm{s}$}

Figure 6. Mechanical properties of the micro parts molded with the two processes.

All the evaluated tensile parameters resulted more dispersed for the parts molded with the usIM than those produced by $\mu \mathrm{IM}$, indicating lower consistency of their mechanical properties. Moreover, no significant effect of the injection speed was observed for both $\mu \mathrm{IM}$ and usIM. 
Fig. 7 shows mass values of molded parts, indicating higher dispersion for usIM parts that led to higher variability of the mechanical properties. This is explained by the lower repeatability and consistency of ultrasound melting in comparison to conventional melting carried out with a reciprocating screw. In general, melting and filling behavior in usIM clearly affect the mechanical properties of the micro parts, thus indicating the need for further clarification about the melting and filling mechanisms.

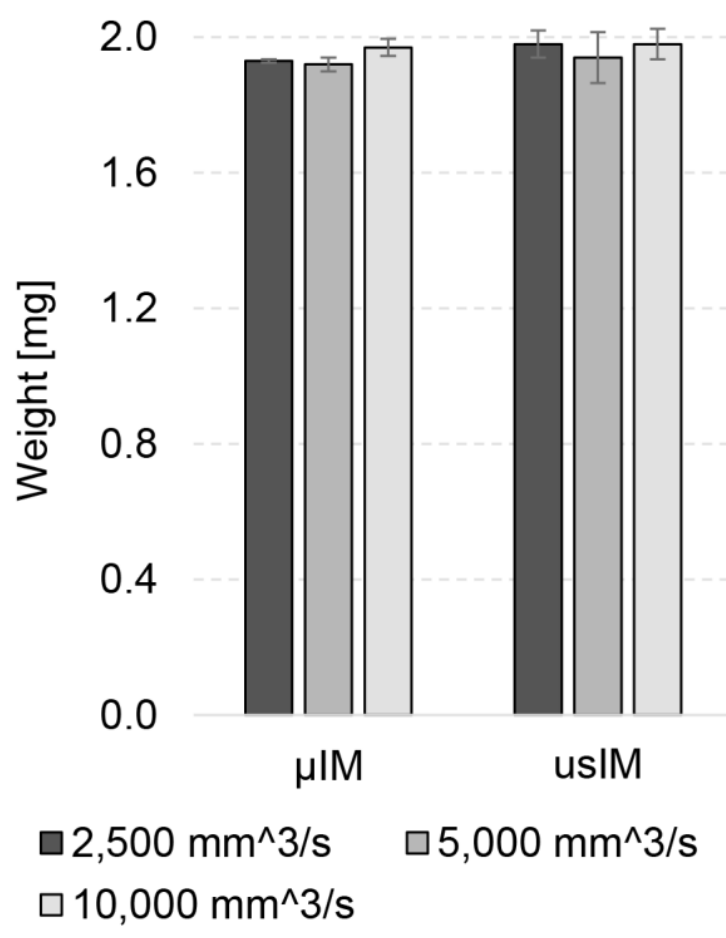

Figure 7. Weight of the micro parts molded with both processes.

\subsection{Crystallinity of the molded parts}

Table 2 reports the results of the DSC characterization performed on parts molded with the two different molding technologies. The results suggest higher crystallization in the usIM parts that are characterized by higher melting enthalpy. In fact, the ratio between the melting enthalpy, measured 
during the first and second heating ramp, is higher for parts molded using the ultrasound melting technology.

The higher level of crystallinity in the usIM samples supports the results of the mechanical characterization. Indeed, the alignment of macromolecular chains in the crystalline structure increases the mechanical properties of the polymer and thus the tensile resistance of the usIM molded samples [32].

Table 2. Results of DSC characterization of the molded micro parts.

\begin{tabular}{ccccccc}
\hline & $\begin{array}{c}\text { Injection } \\
\text { Speed } \\
{[\mathrm{mm} / \mathrm{s}]}\end{array}$ & Mean Val. & Std. Dev. & Mean Val. & Std. Dev. & Melting1/Melting2 \\
& 128 & 77 & 2 & 89 & 3 & 0,87 \\
$\mu \mathrm{IM}$ & 256 & 79 & 1 & 90 & 2 & 0,88 \\
& 512 & 81 & 4 & 91 & 3 & 0,89 \\
& 50 & 90 & 3 & 92 & 3 & 0,98 \\
usIM & 100 & 86 & 4 & 89 & 2 & 0,97 \\
& 200 & 88 & 2 & 91 & 1 & 0,97 \\
\hline
\end{tabular}

\subsection{Flow-induced crystallization}

The morphology of the crystalline structured yielded by the different injection molding technologies was analyzed by overlaying WAXS 2D images for diffraction angles between 0 and $90^{\circ}$. Samples molded using usIM are characterized, for all values of the injection speed, by isotropic scattering due to the formation of spherulites, as shown in Fig. 8 (b). Similar scattering behavior was observed for the hot pressed samples (Fig. 8 (a)), which were obtained under zero-shear conditions. Conversely, $\mu$ IM samples exhibit anisotropic scattering (Fig. 8 (c), (d), (e)) with dominant reflections at plane positions (110), (040) and (130), which are typical of $\alpha$-crystalline phase. Additional diffraction at position (300) indicates the formation of $\beta$-phase. 


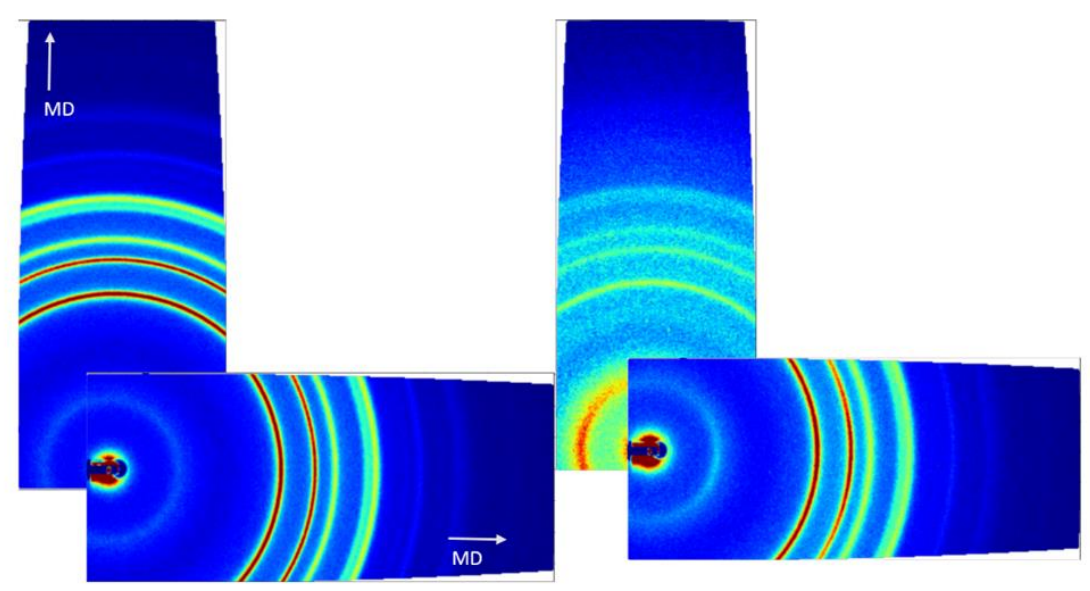

(a)

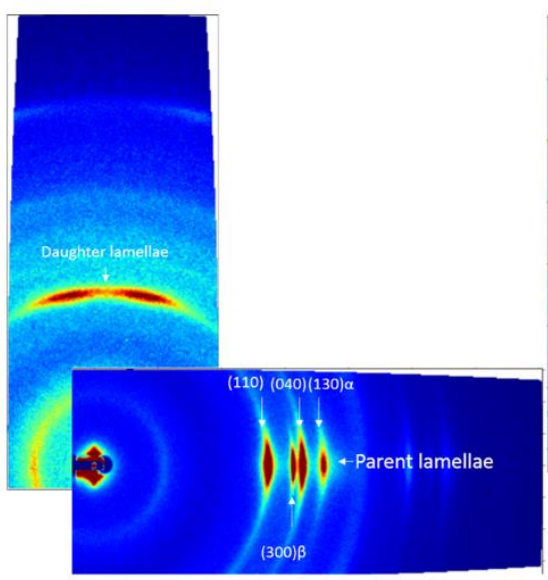

(c)

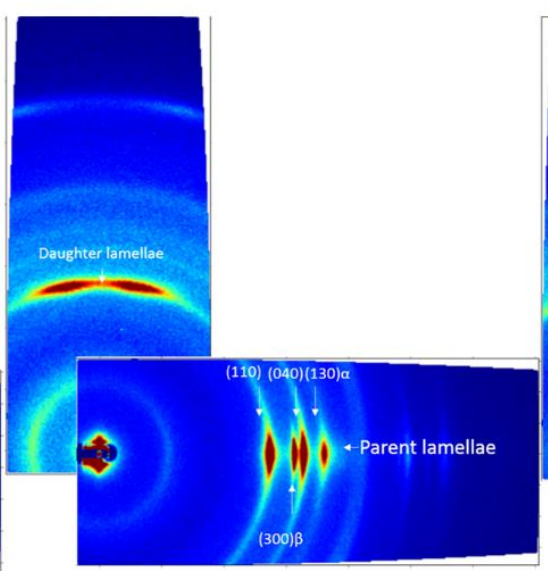

(d) (b)

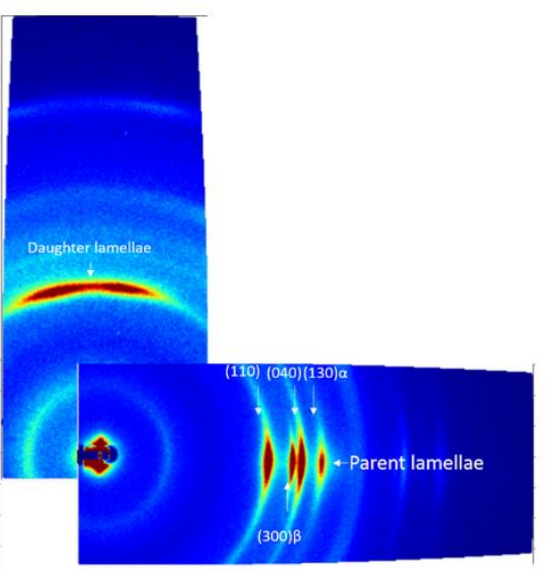

(e)

Figure 8. WAXS scattering patterns for a) hot pressed PP, b) usIM, c) $\mu \mathrm{IM}$ at $2,500 \mathrm{~mm}^{3} / \mathrm{s} \mathrm{d}$ ) $\mu \mathrm{IM}$ at $5,000 \mathrm{~mm}^{3} / \mathrm{s}$ and e) $\mu \mathrm{IM}$ at $10,000 \mathrm{~mm}^{3} / \mathrm{s}$.

Giving a broad azimuthal distribution to plane (110), scattering diffraction in an equatorial direction represents 'parent lamellae', which are crystals growing in the flow direction (c - axis) [30]. Double intensity spots in the meridional direction represent the 'daughter lamellae', which are formed by 'lamellar branching' propagation of the crystal epitaxial growth along the $\mathrm{a}^{*}$ - axis (i.e. perpendicular to 
the $\mathrm{c}$ - axis). The growth in both directions was evaluated considering the relative intensity of reflections in the (110) plane and by applying the Fujiyama method [33]:

$$
\left[a^{*}\right]_{110}=\frac{a^{*}}{C+a^{*}}
$$

where $C$ is the area around the azimuthal angle of $0^{\circ}$ and $a^{*}$ the area around $90^{\circ}$, as shown in Fig. 9.

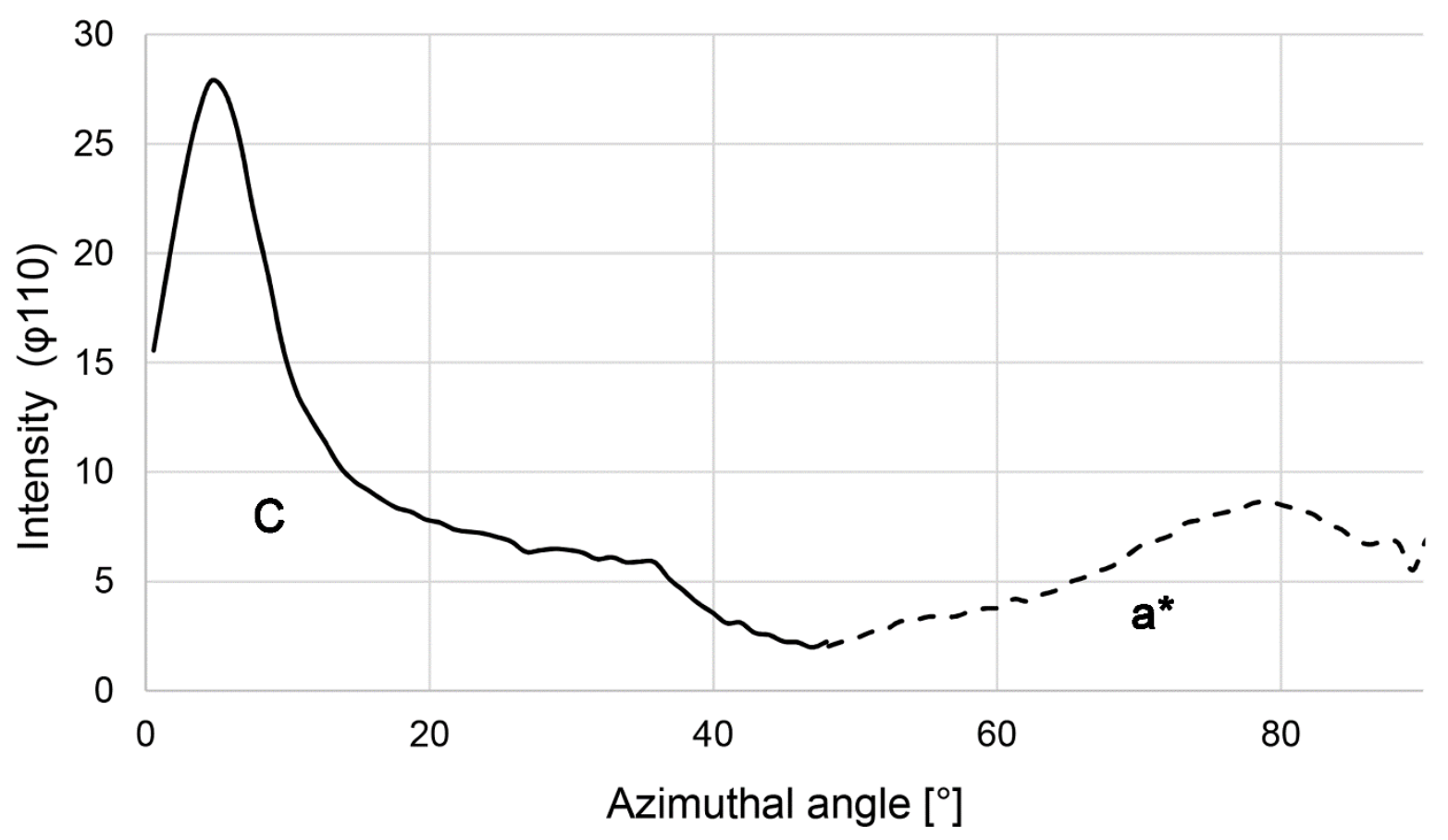

Figure 9. Fujiyama method to determine lamellar branched structure.

Table 3 reports the results of the morphological characterization for the molded micro parts. The fraction $a^{*}$ was found to be zero for the hot-pressed sample and 0.01 for the usIM samples, indicating the absence of 'lamellar branching'. Marked growth of crystals (i.e. high values of $a^{*}$ ) was observed in the $\mu \mathrm{IM}$ samples where higher shear rates characterize the filling of the cavity.

Table 3. Relative $a^{*}$ orientation values calculated for the different samples.

\begin{tabular}{ll}
\hline Sample & {$\left[\mathrm{a}^{*}\right]_{110}$} \\
\hline
\end{tabular}




\begin{tabular}{ll}
\hline hot-pressed & 0.00 \\
usIM & 0.01 \\
$\mu \mathrm{IM} 2,500 \mathrm{~mm}^{3} / \mathrm{s}$ & 0.24 \\
$\mu \mathrm{IM} 5,000 \mathrm{~mm}^{3} / \mathrm{s}$ & 0.35 \\
$\mu \mathrm{IM} 10,000 \mathrm{~mm}^{3} / \mathrm{s}$ & 0.48 \\
\hline
\end{tabular}

Fig. 10 shows SAXS images obtained from the characterization of the different samples. Isotropic scattering in hot-pressed and usIM samples confirm the absence of flow-induced orientation in these samples. Moreover, for the usIM parts no significant effect of higher injection speed was observed. SAXS analysis of $\mu \mathrm{IM}$ parts confirmed scattering diffraction at plane (110) due to the formation of $\alpha$ crystalline phase and meridional high intensity tear drop spots (kebab structure), which originates from the lamellae in the flow direction (shish).

In general, the results of the morphological characterization provide evidence of different filling mechanisms in the usIM and $\mu \mathrm{IM}$ processes. In particular, the comparison of usIM samples with those obtained by hot pressing confirms the very low shear rates during filling. Conversely, higher flowinduced crystallization and higher orientation of the crystal characterize parts obtained by $\mu \mathrm{IM}$, and this effect is increasingly evident for higher $\mu \mathrm{IM}$ injection speed. When using ultrasound melting, the effect of higher injection speed values is not significant, indicating a possible interaction between the melting and filling phases in the usIM process [34]. 


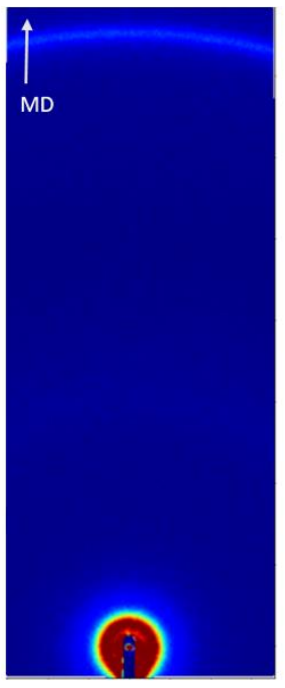

(a)

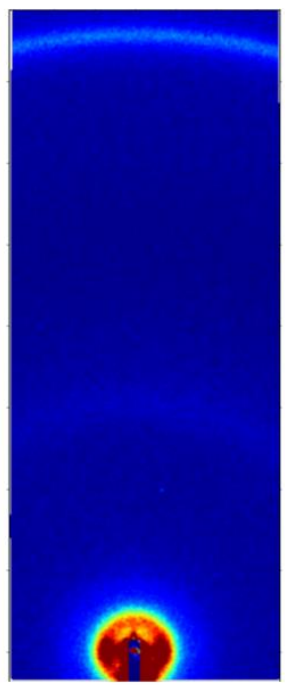

(b)

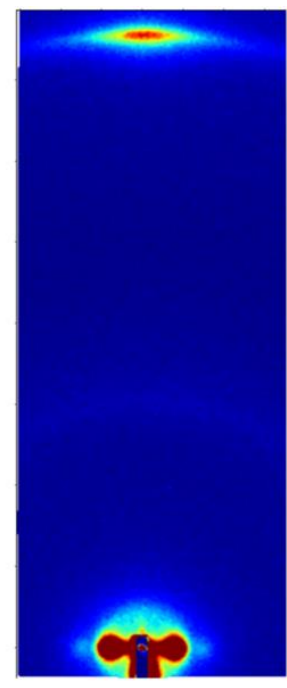

(c)

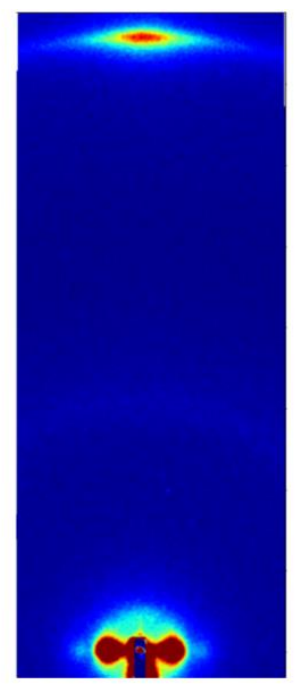

(d)

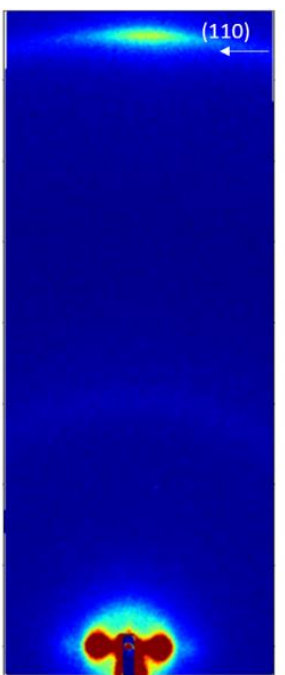

(e)

Figure 10. SAXS results of the tested samples for a) hot pressed PP, b) usIM, c) $\mu \mathrm{IM}$ at $2,500 \mathrm{~mm}^{3} / \mathrm{s}$, d) $\mu \mathrm{IM}$ at $5,000 \mathrm{~mm}^{3} / \mathrm{s}$ and e) $\mu \mathrm{IM} 10,000$ at $\mathrm{mm}^{3} / \mathrm{s}$. MD (flow direction) is vertical.

\section{Results of flow visualization}

\subsection{Cavity filling times}

The effect of ultrasound melting on the speed of the injected polymer melt was evaluated by tracking the coordinates of the advancing flow front from the moment it entered the cavity until it reached its far end. Then, knowing the value of the frame rate used for the acquisition it was possible to link the specific position to its specific time.

Figure 11 reports the results of the flow visualization analysis, showing different trends for the flow front position during cavity filling in the two molding technologies. For the $\mu$ IM process (Fig. 11 (a)), the filling of the tensile bar cavity is characterized by changes of the speed (i.e. slope of the curve) in the three main sections (gate tab, gauge length and end tab). Conversely, for the usIM process no clear distinction is observed between the speed of the polymer in the gauge length and in the end tab (Fig. 11 (b)). 


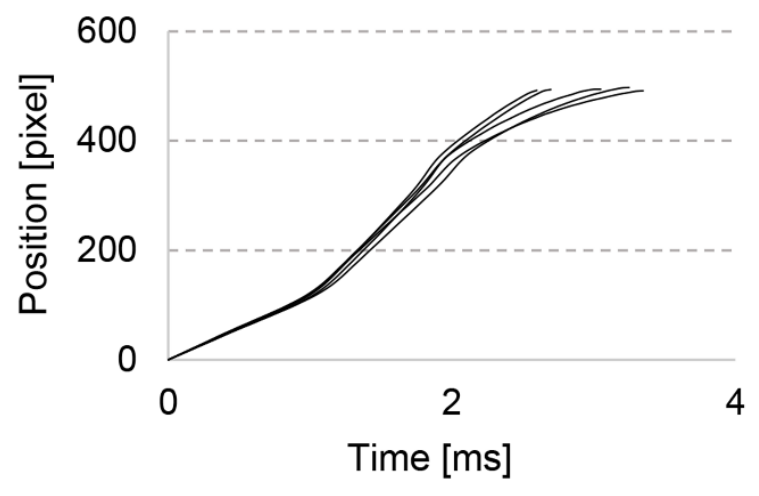

(a)

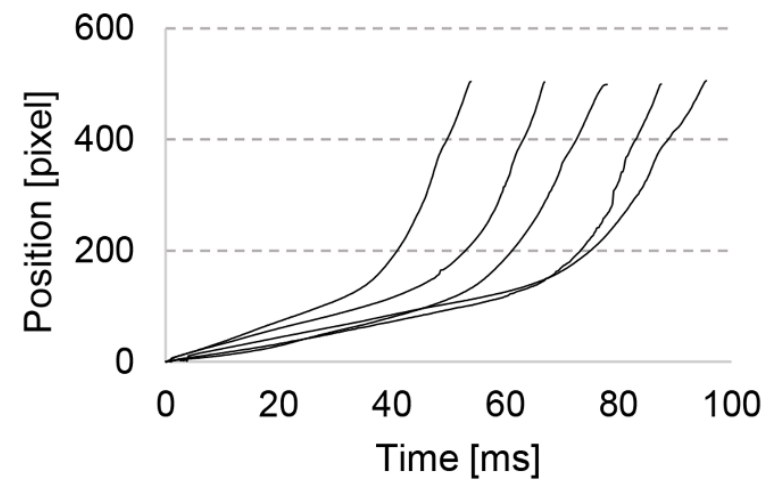

(b)

Figure 11. Flow front tracking for five repeated (a) micro injection molding and (b) ultrasound injection molding experiments for a set flow rate of $5000 \mathrm{~mm}^{3} / \mathrm{s}$. The curves in both plots represent repetitions of experiments performed with the same set of process parameters with the two processes.

The results of the flow front position tracking analysis allowed the evaluation of the filling time for both processes and for the selected values of the injection speed. The times required to fill the cavity are significantly higher for the usIM process compared to $\mu \mathrm{IM}$ (Table 4). Moreover, the values of the filling time calculated are more dispersed, indicating smaller consistency of cavity filling when using ultrasound melting.

Table 4. Filling times obtained from the high-speed camera acquisitions for the processes.

\begin{tabular}{lccc}
\hline & $\begin{array}{c}\text { Filling Time } \\
\text { Injection } \\
\text { Process }\end{array}$ & \multicolumn{2}{c}{$[\mathrm{ms}]$} \\
& & 3.4 & 0.1 \\
Speed $[\mathrm{mm} / \mathrm{s}]$ & Avg. Val. & $\begin{array}{c}\text { Std. } \\
\text { Dev. }\end{array}$ \\
\hline \multirow{2}{*}{ Micro Injection Molding } & 256 & 3.0 & 0.3 \\
& 512 & 1.6 & 0.2 \\
Ultrasound Injection Molding & 50 & 57.5 & 9.6 \\
& 100 & 76.0 & 16.0 \\
& 200 & 70.0 & 12.0 \\
\hline
\end{tabular}




\subsection{Effect of the injection speed}

The filling behavior of the melt polymer in the two processes was also investigated as a function of the injection speed. The effect of higher flow rate resulted in a decrease of the filling time in the $\mu \mathrm{IM}$ process, while no significant variations were observed for the usIM process (Fig. 12). In fact, in the $\mu \mathrm{IM}$ process, the injection plunger exerts its action on a defined amount of polymer, which was previously melted by the mechanical action of a reciprocating screw and then maintained in isothermal conditions. Conversely, in the usIM process, the ultrasonic vibration progressively melts the polymer while the injection plunger moves upwards pushing the material to promote the injection of the molten polymer into the cavity. In these conditions, the injection plunger is acting on a polymer characterized by inhomogeneous thermal and rheological properties. Hence, in the usIM process, the melting and filling phases are simultaneous and they interact. Consequently, the filling time for the usIM process is mainly controlled by the melting rate, which depends on the ultrasound vibration characteristics. 


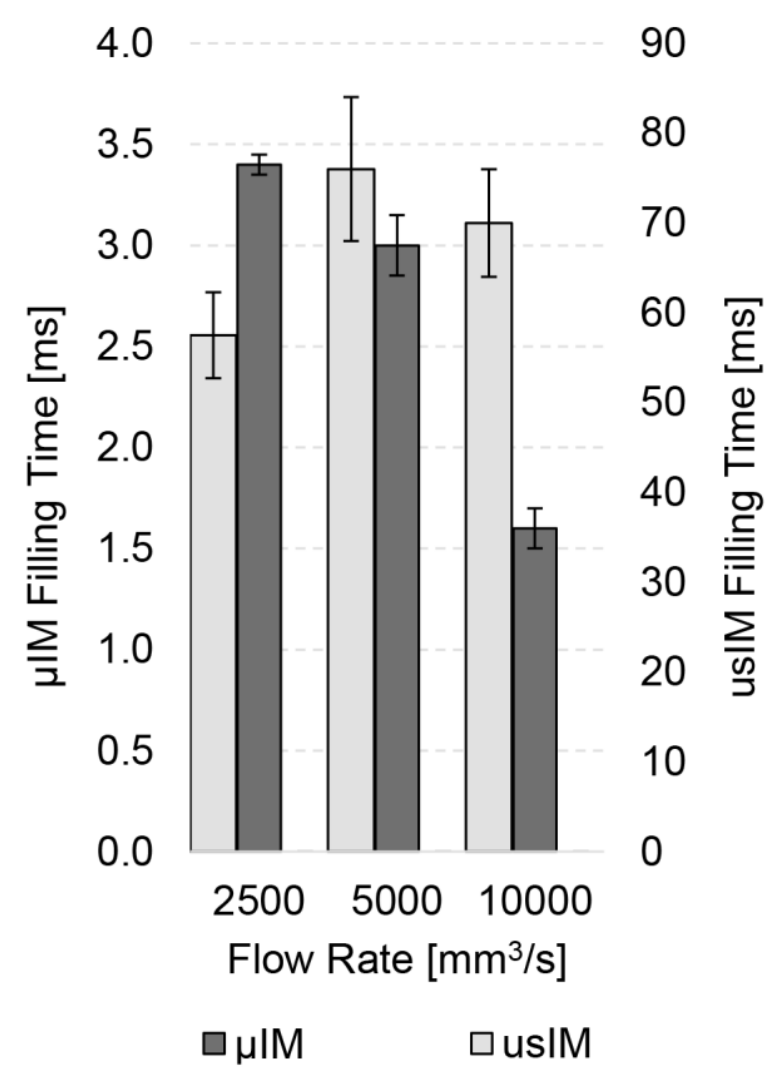

Figure 12. Cavity filling times for both processes as a function of the different set flow rates.

The high-speed camera acquisitions were also used to determine the actual values of the flow front speed during filling of the cavity. The acquired image sequences were processed using NI Vision Assistant only in the gauge section of the cavity, which is characterized by a rectangular cross section $(250 \times 400 \mu \mathrm{m})$. The flow front speed of the injected polymer was determined as the gradient of the advancing flow front position over time and the actual volumetric flow rate was then calculated.

Table 5 reports the calculated flow front speed for the polymer flowing in the gauge length section of the cavity for both processes. The results indicate that the tracked speed of the polymer melt in the cavity is markedly higher for the $\mu \mathrm{IM}$ process compared to the usIM. Moreover, for the latter the effect of changing the injection speed was confirmed to be negligible. 
Table 5. Results of edge detection analysis for the processes and different values of the injection speed.

\begin{tabular}{lccc}
\hline & $\begin{array}{c}\text { Set Flow Rate } \\
{\left[\mathrm{mm}^{3} / \mathrm{s}\right]}\end{array}$ & Flow Front Speed $[\mathrm{mm} / \mathrm{s}]$ \\
Process & & Avg. Val. & Std. Dev. \\
\hline \multirow{2}{*}{ Micro Injection Molding } & 2500 & 2970 & 180 \\
& 5000 & 3834 & 185 \\
& 10000 & 7346 & 705 \\
Ultrasound Injection Molding & 2500 & 259 & 35 \\
& 5000 & 226 & 33 \\
& 10000 & 219 & 17 \\
\hline
\end{tabular}

\subsection{Melt flow temperature}

The temperature of the polymer melt flow during the molding cycle was characterized by means of high-speed thermal imaging, as shown in Fig. 13. Profiles acquired during the $\mu \mathrm{IM}$ cycle are characterized by a steep growth of the temperature during the filling phase and the maximum temperature was acquired at the flow front. Moreover, temperature acquisitions for $\mu \mathrm{IM}$ are characterized by high consistency for acquisitions repeated at constant value of the injection speed. 


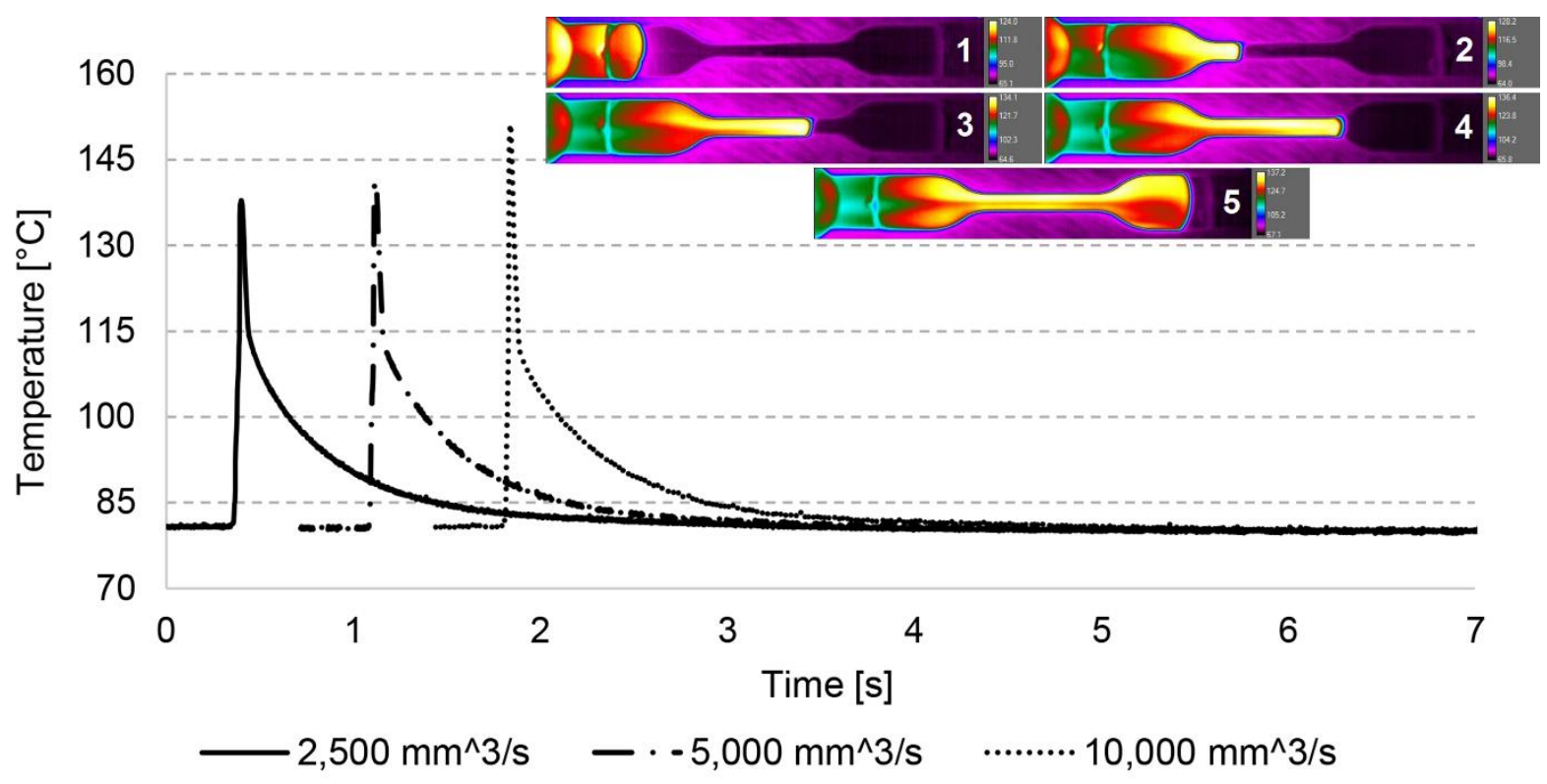

Figure 13. Repeated acquisitions of temperature profiles during $\mu \mathrm{IM}$ cycles at different values of the injection speed.

Conversely, several temperature peaks were observed during the usIM cycle due the different melting and injection mechanisms (Fig. 14 (a)). Indeed, while the ultrasound vibration is maintained (i.e. for 2 seconds), the polymer is progressively melted and injected into the cavity, thus resulting in a temperature distribution within the melt flow that is different from that of conventional $\mu \mathrm{IM}$. Specifically, heat convection from the melt polymer flowing to the cavity lead to higher temperature close to the injection location. Hence, heat is continuously convected through the gate and the polymer is progressively colder moving towards the flow front.

Fig. 14 (b) reports the temperature, the ultrasonic power and the force curves monitored for a single usIM cycle, comparing them with thermal frames. A correlation between the ultrasonic power and the temperature of the polymer melt during filling in the usIM was observed. Specifically, the first material reaches the injection location (frame \#1) just after the first peak of in the ultrasound power profile. The filling of the cavity is accompanied by repeated peaks of both temperature and power, as shown in frames 
$\# 2$, \#3 and \#4. Since, the vibration and the force are maintained after the achievement of the complete filling of the mold cavity, further material flows into the cavity and packing occurs (cf. frame \#5 and \#6).
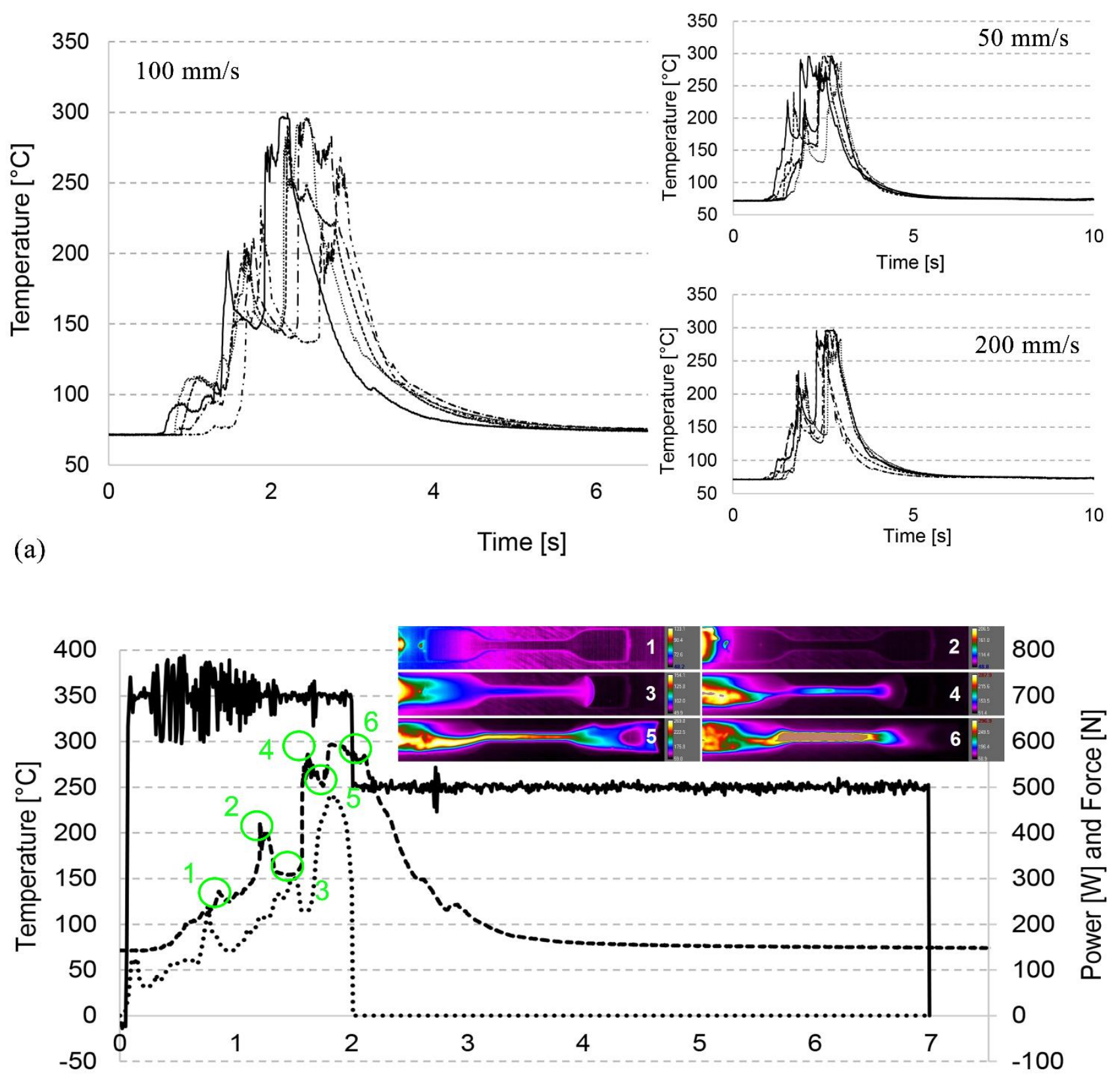

Time (s) 
Figure 14. (a) Repeated acquisitions of temperature profiles during $\mu \mathrm{IM}$ cycles at different values of the injection speed and (b) comparison of temperature, power and force profiles.

Higher values of the injection speed resulted in higher temperature for the $\mu \mathrm{IM}$ process, while no significant variation was observed for the usIM process. Indeed, in the usIM process, the filling of the cavity is controlled by the melting rate, as discussed in Section 5.2.

Fig. 15 reports the maximum values of temperature acquired within the polymer during injection for the two different molding technologies. When using ultrasound vibration to melt the plastic granules, markedly higher temperature peaks were observed. In fact, the heat convected during the progressive melting and injection lead to higher temperature of the polymer melt. Conversely, in $\mu \mathrm{IM}$ the heat convected by the advancing flow front is rapidly dissipated by conduction through the mold surface.

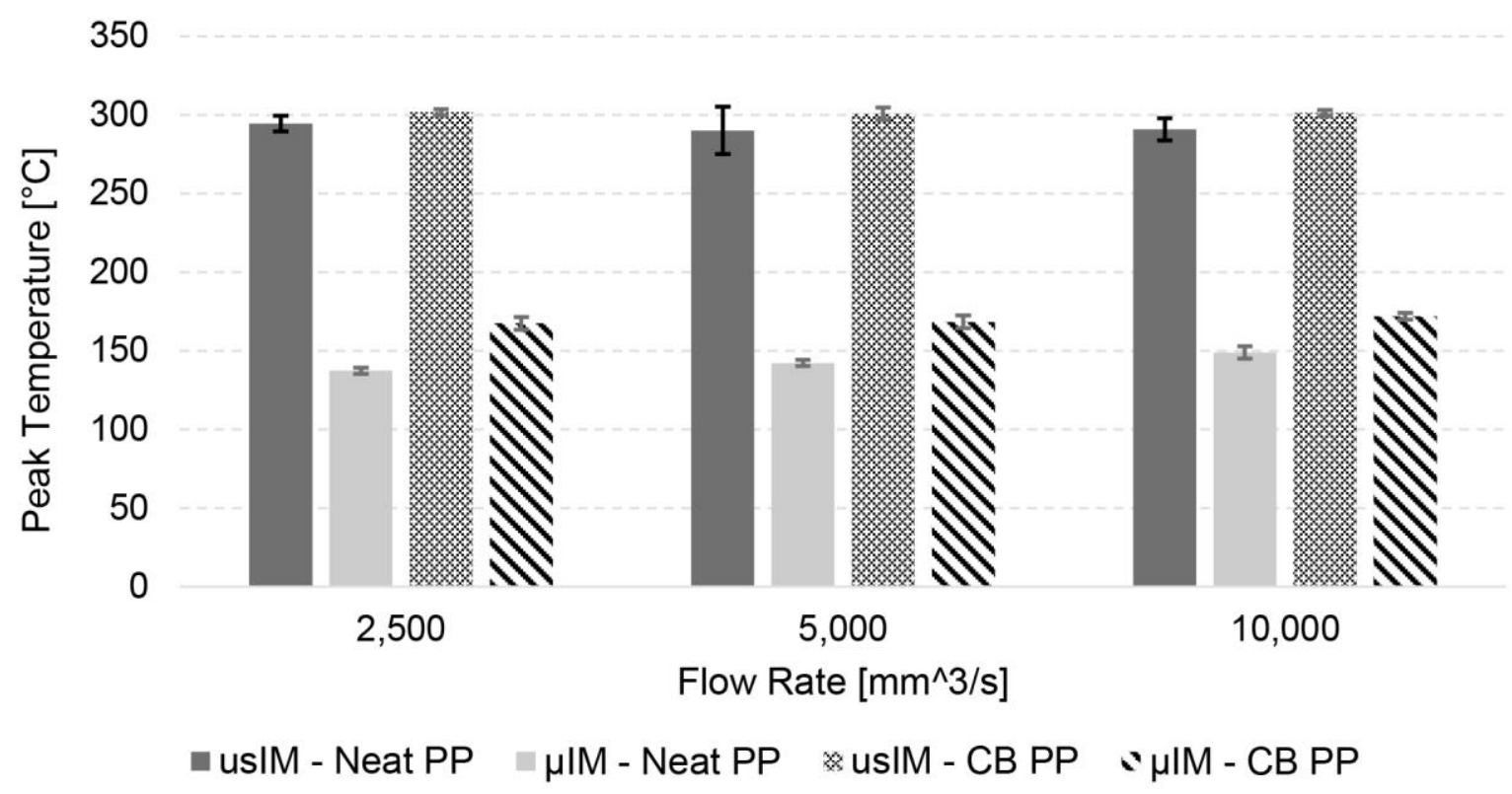

Figure 15. Peak temperatures for the usIM and $\mu \mathrm{IM}$ processes.

\section{Conclusion}



characterization of the effect of ultrasound melting on the mechanical properties of polypropylene micro parts. A comprehensive characterization of the filling behavior of the two processes was carried out by means of high-speed thermal and conventional direct flow visualization.

The tensile properties of the molded micro parts indicated that the overall mechanical properties achieved with the usIM process are not just comparable to those obtained with the $\mu \mathrm{IM}$, but offer significantly improved performance. However, the smaller repeatability in the mechanical behavior of the parts molded with usIM are due to the higher weight variability.

The results of the tracking of the flow front positions showed that $\mu \mathrm{IM}$ is characterized by a more consistent and repeatable filling behavior compared to usIM. In particular, in the conventional process increasing the injection speed resulted in significantly higher flow front speed, while the usIM had no significant effect on increasing the flow rate. This different response to the change of the set flow rate can be reconducted to the different melting mechanisms. In fact, compared to the conventional melting, which is carried out with a reciprocating screw, the use of ultrasound vibration leads to inhomogeneous thermal and rheological conditions of the polymer melt.

Moreover, the ultrasound melting resulted in higher temperature of the injected polymer melt due to the sustainment of the ultrasound vibration during filling and packing phases. Indeed, several temperature peaks were observed close to the injection location and not on the flow front as in the conventional $\mu \mathrm{IM}$ process. 


\section{Funding}

This research did not receive any specific grant from funding agencies in the public, commercial, or not-for-profit sectors. 


\section{References}

[1] Attia, U. M., Marson, S., \& Alcock, J. R. (2009). Micro-injection moulding of polymer microfluidic devices. Microfluidics and Nanofluidics, 7(1), 1-28.

[2] Tseng, S. C., Chen, Y. C., Kuo, C. L., \& Shew, B. Y. (2005). A study of integration of LIGA and M-EDM technology on the microinjection molding of ink-jet printers' nozzle plates. Microsystem technologies, 12(1-2), 116-119.

[3] Angelov, A. K., \& Coulter, J. P. (2008). The development and characterization of polymer microinjection molded gratings. Polymer Engineering \& Science, 48(11), 2169-2177.

[4] Wallrabe, U., Dittrich, H., Friedsam, G., Hanemann, T., Mohr, J., Müller, K., ... \& Zißler, W. (2002). Micromolded easy-assembly multi fiber connector: RibCon®. Microsystem technologies, 8(23), 83-87.

[5] Lucchetta, G., Sorgato, M., Zanchetta, E., Brusatin, G., Guidi, E., Di Liddo, R., \& Conconi, M. T. (2015). Effect of injection molded micro-structured polystyrene surfaces on proliferation of mc3t3-e1 cells. Express Polym Lett, 9(4), 354-361.

[6] Masato, D., Sorgato, M., Babenko, M., Whiteside, B., \& Lucchetta, G. (2017). Thin-wall injection molding of polystyrene parts with coated and uncoated cavities. Materials \& Design, 141, 286-295.

[7] Masato, D., Sorgato, M., Parenti, P., Annoni, M., \& Lucchetta, G. (2017). Impact of deep cores surface topography generated by micro milling on the demolding force in micro injection molding. Journal of Materials Processing Technology, 246, 211-223.

[8] Yang, C., Yin, X. H., \& Cheng, G. M. (2013). Microinjection molding of microsystem components: new aspects in improving performance. Journal of Micromechanics and Microengineering, 23(9), 093001. 
[9] Whiteside, B. R., Martyn, M. T., Coates, P. D., Allan, P. S., Hornsby, P. R., \& Greenway, G. (2003). Micromoulding: process characteristics and product properties. Plastics, rubber and composites, 32(6), 231-239.

[10] Hansen, H. N., Hocken, R. J., \& Tosello, G. (2011). Replication of micro and nano surface geometries. CIRP Annals-Manufacturing Technology, 60(2), 695-714.

[11] Sorgato, M., Masato, D., \& Lucchetta, G. (2016). Effect of vacuum venting and mold wettability on the replication of micro-structured surfaces. Microsystem Technologies, 1-10.

[12] Masato, D., Sorgato, M., \& Lucchetta, G. (2016). Analysis of the influence of part thickness on the replication of micro-structured surfaces by injection molding. Materials \& Design, 95, 219-224. [13] Sorgato, M., Babenko, M., Lucchetta, G., \& Whiteside, B. (2017). Investigation of the influence of vacuum venting on mould surface temperature in micro injection moulding. The International Journal of Advanced Manufacturing Technology, 88(1-4), 547-555.

[14] Lucchetta, G., Sorgato, M., Carmignato, S., \& Savio, E. (2014). Investigating the technological limits of micro-injection molding in replicating high aspect ratio micro-structured surfaces. CIRP Annals-Manufacturing Technology, 63(1), 521-524.

[15] Masato, D., Rathore, J., Sorgato, M., Carmignato, S., \& Lucchetta, G. (2017). Analysis of the shrinkage of injection-molded fiber-reinforced thin-wall parts. Materials \& Design, 132, 496-504. [16] Lucchetta, G., Masato, D., Sorgato, M., Crema, L., \& Savio, E. (2016). Effects of different mould coatings on polymer filling flow in thin-wall injection moulding. CIRP Annals-Manufacturing Technology, 65(1), 537-540.

[17] Giboz, J., Copponnex, T., \& Mélé, P. (2009). Microinjection molding of thermoplastic polymers: morphological comparison with conventional injection molding. Journal of Micromechanics and Microengineering, 19(2), 025023. 
[18] Michaeli, W., Spennemann, A., \& Gärtner, R. (2002). New plastification concepts for micro injection moulding. Microsystem technologies, 8(1), 55-57.

[19] Michaeli, W., Kamps, T., \& Hopmann, C. (2011). Manufacturing of polymer micro parts by ultrasonic plasticization and direct injection. Microsystem Technologies, 17(2), 243-249.

[20] Wu, W., Peng, H., Jia, Y., \& Jiang, B. (2016). Characteristics and mechanisms of polymer interfacial friction heating in ultrasonic plasticization for micro injection molding. Microsystem Technologies, 1-8.

[21] Jiang, B., Peng, H., Wu, W., Jia, Y., \& Zhang, Y. (2016). Numerical Simulation and Experimental Investigation of the Viscoelastic Heating Mechanism in Ultrasonic Plasticizing of Amorphous Polymers for Micro Injection Molding. Polymers, 8(5), 199.

[22] Chen, G., Guo, S., \& Li, H. (2002). Ultrasonic improvement of rheological behavior of polystyrene. Journal of applied polymer science, 84(13), 2451-2460.

[23] Chen, J., Chen, Y., Li, H., Lai, S. Y., \& Jow, J. (2010). Physical and chemical effects of ultrasound vibration on polymer melt in extrusion. Ultrasonics sonochemistry, 17(1), 66-71. [24] Ávila- Orta, C., Espinoza- González, C., Martínez- Colunga, G., Bueno- Baqués, D., Maffezzoli, A., \& Lionetto, F. (2013). An overview of progress and current challenges in ultrasonic treatment of polymer melts. Advances in Polymer Technology, 32(S1), E582-E602.

[25] Sacristan, M., Planta, X., Morell, M., \& Puiggali, J. (2014). Effects of ultrasonic vibration on the micro-molding processing of polylactide. Ultrasonics sonochemistry, 21(1), 376-386.

[26] Grabalosa, J., Ferrer, I., Elías-Zúñiga, A., \& Ciurana, J. (2016). Influence of processing conditions on manufacturing polyamide parts by ultrasonic molding. Materials \& Design, 98, 20-30. [27] Michaeli, W., \& Starke, C. (2005). Ultrasonic investigations of the thermoplastics injection moulding process. Polymer testing, 24(2), 205-209. 
[28] Negre Gubau, P., Grabalosa Saubí, J., Ferrer Real, I., \& Ciurana, Q. D. (2015). Study of the ultrasonic molding process parameters for manufacturing polypropylene parts. Procedia Engineering, 2015, vol. 32, p. 7-14.

[29] Whiteside, B. R., Spares, R., Brown, E. C., Norris, K., Coates, P. D., Kobayashi, M., ... \& Cheng, C. C. (2008). Optical imaging metrology for micromoulding cavity flows and products. Plastics, Rubber and Composites, 37(2-4), 57-66.

[30] Ma, Z., Balzano, L., Portale, G., \& Peters, G. W. (2014). Flow induced crystallization in isotactic polypropylene during and after flow. Polymer, 55(23), 6140-6151.

[31] Babenko, M., Sweeney, J., Petkov, P., Lacan, F., Bigot, S., \& Whiteside, B. (2017). Evaluation of Heat Transfer at the Cavity-Polymer Interface in Microinjection Moulding based on Experimental and Simulation Study. Applied Thermal Engineering.

[32] Fischer, C., \& Drummer, D. (2016). Crystallization and mechanical properties of polypropylene under processing-relevant cooling conditions with respect to isothermal holding time. International Journal of Polymer Science, 2016.

[33] Schrauwen, B. A., Janssen, R. P., Govaert, L. E., \& Meijer, H. E. (2004). Intrinsic deformation behavior of semicrystalline polymers. Macromolecules, 37(16), 6069-6078.

[34] Gao, S., Qiu, Z., Ma, Z., \& Yang, Y. (2017). Flow properties of polymer melt in longitudinal ultrasonic- assisted microinjection molding. Polymer Engineering \& Science, 57(8), 797-805. 


\section{Figure captions}

Figure 1. Design of the molded micro part. All dimensions are in millimeters.

Figure 2. Main phases of the usIM process, (a) pellet feeding and compression, (b) melting, (c) injection.

Figure 3. Mold design, as mounted on the usIM machine, and indication of its main components.

Figure 4. Procedure adopted for the DSC thermal characterization of the injection molded samples.

Figure 5. Edge detection of the flow front position on the acquired frame.

Figure 6. Mechanical properties of the micro parts molded with the two processes.

Figure 7. Weight of the micro parts molded with both processes.

Figure 8. WAXS scattering patterns for a) hot pressed PP, b) usIM, c) $\mu \mathrm{IM}$ at 2,500 $\mathrm{mm} 3 / \mathrm{s} \mathrm{d}$ ) $\mu \mathrm{IM}$ at 5,000 $\mathrm{mm} 3 / \mathrm{s}$ and e) $\mu \mathrm{IM}$ at $10,000 \mathrm{~mm} 3 / \mathrm{s}$.

Figure 9. Fujiyama method to determine lamellar branched structure.

Figure 10. SAXS results of the tested samples for a) hot pressed PP, b) usIM, c) $\mu \mathrm{IM}$ at $2,500 \mathrm{~mm} 3 / \mathrm{s}, \mathrm{d}) \mu \mathrm{IM}$ at $5,000 \mathrm{~mm} 3 / \mathrm{s}$ and e) $\mu \mathrm{IM} 10,000$ at $\mathrm{mm} 3 / \mathrm{s}$. MD (flow direction) is vertical.

Figure 11. Flow front tracking for five repeated (a) micro injection molding and (b) ultrasound injection molding experiments for a set flow rate of $5000 \mathrm{~mm} 3 / \mathrm{s}$. The curves in both plots represent repetitions of experiments performed with the same set of process parameters with the two processes.

Figure 12. Cavity filling times for both processes as a function of the different set flow rates.

Figure 13. Repeated acquisitions of temperature profiles during $\mu \mathrm{IM}$ cycles at different values of the injection speed.

Figure 14. (a) Repeated acquisitions of temperature profiles during $\mu \mathrm{IM}$ cycles at different values of the injection speed and (b) comparison of temperature, power and force profiles.

Figure 15. Peak temperatures for the usIM and $\mu \mathrm{IM}$ processes. 


\section{Table captions}

Table 1. Main properties of the polymer selected for the molding experiments.

Table 2. Results of DSC characterization of the molded micro parts.

Table 3. Relative a* orientation values calculated for the different samples.

Table 4. Filling times obtained from the high-speed camera acquisitions for the processes.

Table 5. Results of edge detection analysis for the processes and different values of the injection speed. 